\title{
Desoxypipradrol - eine neue (alte) Designerdroge
}

\section{Eine Fallserie}

D. Müller, V. Angerer, V. Auwärter, H. Neurath, G. Liebetrau, S. Just, J. Kithinji, M. Hermanns-Clausen

Neue psychoaktive Substanzen, sogenannte „Legal Highs“, können problemlos über das Internet gekauft werden und werden daher zunehmend konsumiert. Auf den Produkten befinden sich keine Angaben zur Wirkstoffzusammensetzung und -konzentration. Hier berichten wir über sechs Notfallpatienten mit Vergiftungen, bei denen Desoxypipradrol im Serum und Urin nachgewiesen wurde.

\section{4-Jähriger mit akutem Nierenversagen}

Anamnese und klinischer Befund | Drei Tage nach Einnahme einer „Teezubereitung“ wird ein 24-jähriger Patient aufgrund eines akuten Nierenversagens vorgestellt. Er glaubte, Methylendioxypyrovaleron eingenommen zu haben. Es bestehen folgende Befunde:

- Exsikkose

- initiale Agitiertheit/Verwirrtheit

- Mydriasis

- Tachykardie (120/min)

- starke Muskelschmerzen

- Perimyokarditis

- Pneumonie

- Glasgow Coma Score von 3

- initiale Körpertemperatur von $37,8^{\circ} \mathrm{C}$

- anamnestisches Fieber

- Rhabdomyolyse

Toxikologische Analyse I Wir untersuchten Urinbzw. Serumproben mit

- LC-MS ${ }^{\mathrm{n}}$ Ionenfallenscreening mittels Toxtyper ${ }^{\mathrm{TM}}$ und

- einem Drogenscreening mittels Immunoassays, das im Falle positiver Befunde mit GC-MS oder LC-MS / MS überprüft wurde.

Zusätzlich erfolgten spezielle LC-MS/MS-Analysen zur Erfassung von NPS und deren Metaboliten.

Toxikologischer Befund | Desoxypipradrol war im Urin in einer Konzentration von $2300 \mathrm{ng} / \mathrm{ml}$, in der Serumprobe nicht (mehr) nachweisbar. Im Serum fanden sich

- $38 \mathrm{ng} / \mathrm{ml}$ Amfetamin

- $15 \mathrm{ng} / \mathrm{ml}$ Benzoylecgonin

- 5,5 ng/ml Clonazepam

Gleichzeitig wurden im Urin folgende Substanzen nachgewiesen:

- $620 \mathrm{ng} / \mathrm{ml}$ Amfetamin

- $94 \mathrm{ng} / \mathrm{ml}$ Morphin

- $8,1 \mathrm{ng} / \mathrm{ml}$ Codein

- $10 \mathrm{ng} / \mathrm{ml}$ Metamfetamin

- 2,0 ng/ml Ephedrin

- $16 \mathrm{ng} / \mathrm{ml}$ Norephedrin

- $13 \mathrm{ng} / \mathrm{ml}$ Tetrahydrocannabinol-COOH
Blutwerte

- Kreatin-Kinase: 75000U/1 (<170), nach 2 Tagen $15700 \mathrm{U} / 1$

- Myoglobin: $36000 \mathrm{mg} / \mathrm{l}(<55)$, nach 2 Tagen $2600 \mathrm{mg} / \mathrm{L}$

- Kreatinin: 2,0 (<1,1), nach 2 Tagen 4,5 mg/dl

- GOT initial: $2260 \mathrm{U} / 1$

- GPT: $900 \mathrm{U} / \mathrm{l}$

- GGT: $166 \mathrm{U} / 1$

\section{7-Jährige mit „Kreislaufproblemen“}

Anamnese und klinischer Befund | Eine 17-jährige Patientin wurde vorgestellt, nachdem sie sogenanntes „Badesalz“ nasal aufgenommen hatte.

Sie litt unter

- Verwirrtheit

- ausgeprägter motorischer Unruhe

- Tachypnoe

- Angstgefühl

- Tremor

- „Kreislaufproblemen“

- Tachykardie (142/min)

- Exsikkose

- Temperaturerhöhung $\left(37,8^{\circ} \mathrm{C}\right)$

- einer Rhabdomyolyse

\section{Blutwerte}

- Kreatin-Kinase: 644 U/1 $(<170)$

- Kreatinin: zunächst $1,03 \mathrm{mg} / \mathrm{dl}(<1,09)$, dann Anstieg auf $1,12 \mathrm{mg} / \mathrm{dl}$, nach Volumengabe $0,78 \mathrm{mg} / \mathrm{dl}$

Toxikologischer Befund I Die Desoxypipradrolkonzentration im Serum betrug $150 \mathrm{ng} / \mathrm{ml}$ und in der Urinproben $1800 \mathrm{ng} / \mathrm{ml}$. Außerdem wurden im Serum nachgewiesen:

- 35,8 ng/ml Amfetamin

- 3,4 ng/ml MDMA

Im Urin fanden sich

- $250 \mathrm{ng} / \mathrm{ml}$ Amfetamin sowie

- $180 \mathrm{ng} / \mathrm{ml}$ MDMA. 
Abb. 1 Stukturformeln von Amfetamin (A), Methylphenidat (B), Pipradol (C) und Desoxypipradrol (D). *chirales Zentrum.<smiles>CC(N)Cc1ccccc1</smiles>

A<smiles>COC(=O)C(c1ccccc1)C1CCCCN1</smiles>

B<smiles>OC(c1ccccc1)(c1ccccc1)C1CCCCN1</smiles>

C<smiles>c1ccc(C(c2ccccc2)C2CCCCN2)cc1</smiles>

D

\section{7-Jähriger mit Taubheitsgefühlen}

Anamnese und klinischer Befund | Ein 17-jähriger Patient stellte sich nach nasaler Aufnahme von $50 \mathrm{mg}$ Desoxypipradrol vor. Er berichtete über Taubheitsgefühl und Parästhesien des rechten Armes und der Füße, Tachykardie, vermehrtes Schwitzen sowie ein ausgeprägtes Unruhegefühl und Schlafstörungen. Die Herzfrequenz und der Blutdruck wurden mit 110/min bzw. 135/64 mm Hg gemessen, außerdem lag eine Mydriasis vor. Die Kreatinkinase wurde mit 397 U/1 bestimmt. Der Patient erlitt eine Rhabdomyolyse.

Toxikologischer Befund I Im Serum nachwiesen wurden:

- $120 \mathrm{ng} / \mathrm{ml}$ Desoxypipradrol

- $44 \mathrm{ng} / \mathrm{ml}$ Amfetamin

- 4,2 ng/ml MDMA

- $1,5 \mathrm{ng} / \mathrm{ml}$ MDA.

Im Urin fanden sich

- $2200 \mathrm{ng} / \mathrm{ml}$ Desoxypipradrol,

- $250 \mathrm{ng} / \mathrm{ml}$ Amfetamin.

- $1020 \mathrm{ng} / \mathrm{ml}$ MDMA,

- 1,5 ng/ml MDA und

- Nikotin-Metabolite.

\section{4-Jähriger mit Verdacht auf Leberversagen}

Verena Angerer ist Doktorandin am Institut für Rechtsmedizin / Forensische Toxikologie, Universitätsklinikum Freiburg. verena.angerer@ uniklinik-freiburg.de

Josephine Kithinji ist Beratungsärztin in der Klinik für Allgemein- und Jugendmedizin, Universitätsklinikum Freiburg. josephine.kithinji@ uniklinik-freiburg.de

Prof. Dr. Volker Auwärter ist Laborleiter am Institut für Rechtsmedizin / Forensische Toxikologie, Universitätsklinikum Freiburg. volker.auwaerter@ uniklinik-freiburg.de
Anamnese und klinischer Befund | Ein 24-jähriger Patient wurde mit Verdacht auf Leberversagen vorgestellt. Er berichtete über dunklen Urin, die weitere Anamnese war nicht sicher zu erheben. Die Herzfrequenz und der Blutdruck wurden mit 112/ min bzw. 134/ 87 mm Hg gemessen. Auch bei diesem Patienten kam es zu einer Rhabdomyolyse.

\section{Blutwerte}

- Kreatinkinase: 170208 U/ml (<170) am Aufnahmetag, nach 3 Tagen $17666 \mathrm{U} / \mathrm{ml}$

- AST: 2108 U/l (<35), nach 3 Tagen 507 U/ml

- ALT: $410 \mathrm{U} / \mathrm{l}(<45)$ nach 3 Tagen $230 \mathrm{U} / \mathrm{ml}$

- S-Kreatinin $114 \mu \mathrm{mol} / \mathrm{l}(50-104)$, nach 3 Tagen $93 \mu \mathrm{mol} / 1$

Toxikologischer Befund | Im Urin konnten folgende Substanzen nachgewiesen werden:

- $5900 \mathrm{ng} / \mathrm{ml}$ Desoxypipradrol

- $440 \mathrm{ng} / \mathrm{ml}$ Methoxyphenidin

- $120 \mathrm{ng} / \mathrm{ml}$ Methylon

\section{Junger Mann mit Hyperhidrosis und Erbrechen}

Klinischer Befund | Ein junger, erwachsener Mann wurde mit ausgeprägter Vigilanzminderung, Tachykardie, Hypertonie $(210 / 90 \mathrm{~mm} \mathrm{Hg})$, Erbrechen und Hyperhidrosis vorgestellt.

Toxikologischer Befund I Im Serum wurden $84 \mathrm{ng} / \mathrm{ml}$ Desoxypipradrol, 0,26\%。 Ethanol und $<0,1 \mathrm{ng} / \mathrm{ml} 5 \mathrm{~F}-\mathrm{PB}-22$ nachgewiesen. In seinem Urin fanden sich:

- $440 \mathrm{ng} / \mathrm{ml}$ Methoxyphenidin

- $120 \mathrm{ng} / \mathrm{ml}$ Methylon

- 2,4 ng/ml bk-MDDA (Dimethylon)

- qualitativ: Metamfetamin, Pyrazolam, Nikotin und Koffein

\section{0-Jähriger mit „Kribbelgefühl“}

Klinischer Befund | Ein 30-jähriger Patient wurde vorgestellt mit

- Unruhe

- Hypertonie

- Thoraxschmerz und

- Paraesthesien.

Toxikologischer Befund I Die Desoxypipradrolkonzentration im Urin betrug 1,5 ng/ml. Zusätzlich wurden Metaboliten von AM-2201, 5F-PB22, Tetrahydrocannabinol, Koffein und Nikotin nachgewiesen.

\section{Diskussion}

Altbekannte Wirkstoffe, unbekannte Dosis | Frei verkäufliche NPS enthalten bisweilen altbekannte Wirkstoffe, die aus der pharmazeutischen Entwicklung stammen oder auch als Pharmakon eingesetzt (und wieder vom Markt genommen) worden sind. Eine Deklaration der Inhaltsstoffe mit Hinweisen bezüglich der Dosierung erfolgt auf den Produkten in der Regel nicht. Eine Ausnahme stellen die sogenannten „Research Chemicals" dar. Dem Konsumenten bleibt somit meist verborgen, ob er einzelne Wirkstoffe oder Gemische zu sich nimmt, die mit gleichzeitig konsumierten anderen Produkten oder einer bestehenden Pharmakotherapie zu Wechselwirkungen führen können. Die Abschätzung einer wirksa- 
men Dosis ist abgesehen von „Research Chemicals“ nicht möglich und erfordert insbesondere bei den potenteren Wirkstoffen den Einsatz einer Feinwaage, sodass es sehr leicht zu erheblichen Überdosierungen kommen kann.

Stärker als Amfetamine I In den 1950er Jahren wurde Desoxypipradrol (2-Diphenylmethylpiperidin, 2-DPMP, > Abb. 1 D) [1] zur Behandlung der Narkolepsie bzw. der Aufmerksamkeitsdefizit-/ Hyperaktivitätsstörung (ADHS) beschrieben einige Jahre nach Methylphenidat (Ritalin, - Abb. 1B) und Pipradrol (Schlankheitsmittel [2], - Abb. 1C). Der Norepinephrin-Dopamin-Wiederaufnahme-Hemmer zeichnet sich durch eine sehr lange Wirkdauer sowie eine stärkere Wirksamkeit im Vergleich zu Amfetamin ( $\triangleright$ Abb. 1A) aus [3]. In Europa wurde er erstmals im Jahr 2007 in Großbritannien als „Legal High“ nachgewiesen $[4,5]$. Die Substanz wird in den gebräuchlichen Drogenschnelltests nicht erfasst und ist in Deutschland bisher nicht reguliert.

Rhabdomyolyse nach Konsum | Rhabdomyolysen nach Konsum von Desoxypipradrol-haltigen Drogenmischungen wurden häufig beschrieben [6]. Bislang wurde jedoch noch kein Zusammenhang zwischen der klinischen Symptomatik und den Wirkstoffkonzentrationen in Serum oder Urin hergestellt. Bei drei Patienten, deren Desoxypipradrol-Urinkonzentration bei gering erhöhter Kreatininkonzentration auf eine sehr hohe aufgenommene Dosis hindeutet, trat eine Rhabdomyolyse auf. Als Ursache der Rhabdomyolyse ist eine toxische Ursache oder ein Krampfanfall (für den keine anamnestischen Hinweise bestehen) zu diskutieren.

Multipler Substanzgebrauch | Eine toxische Ursache kommt insbesondere bei multiplem Substanzgebrauch in Betracht. Dieser wurde bei allen Patienten nachgewiesen. Vor allem bei der Kombination mehrerer serotonerg wirkender Drogen oder Pharmaka sollte eine massive Stimulation der 5- $\mathrm{HT}_{2 \mathrm{~A}}$-Rezeptoren (oder der 5-HT $\mathrm{HA}_{1 \mathrm{~A}}$-Rezeptoren) mit der Folge eines Serotoninsyndroms in Betracht gezogen werden [7]. Die AmfetaminSerumkonzentration lag bei drei Patienten im unteren „therapeutischen Bereich“ (20-100 $\mathrm{ng} / \mathrm{ml}$ ). Die Serumkonzentrationen von MDMA und MDA waren niedrig. In einem Fall wurde aufgrund des Anfangsverdachts auf ein Leberversagen die Rhabdomyolyse verspätet festgestellt. Hier wurde kein Amfetamin nachgewiesen.

Mortalität | Der Konsum von scheinbar harmlosen NPS kann zu bedrohlichen Vergiftungen mit protrahiertem Verlauf führen. Es wurden einzelne Todesfälle mit postmortalem Nachweis von Desoxypipradrol beschrieben $[8,9]$, wobei zwischen dem Konsum von Desoxypipradol und dem Todeseintritt kein klarer kausaler Zusammenhang hergestellt werden konnte.

\section{Konsequenz für Klinik und Praxis}

- Der Konsum von neuen psychoaktiven Substanzen (NPS) hat in den letzten Jahren erheblich zugenommen.

- Unerwünschte Begleitwirkungen erfordern häufiger eine medizinische Behandlung.

- Bei Überdosierungen, gleichzeitigem Konsum verschiedener Wirkstoffe oder bei bestehender Vormedikation können synergistische Interaktionen auftreten.

- Nach Aufnahme von Desoxypipradrol in Kombination mit anderen NPS kann sich Rhabdomyolyse entwickeln.

\section{Danksagung}

Diese Publikation wurde durch die finanzielle Unterstützung der EU-Kommission (DG Justice, JUST/2011/DPIP/AG/3597), des Bundesministeriums für Gesundheit und der Stadt Frankfurt a. M., Drogenreferat, ermöglicht.

\section{Literatur}

1 Baselt RC. Disposition of toxic drugs and chemicals in man. 14. Aufl. Seal Beach, Ca: Biomedical Publications; 2014: 2172-2173

2 Gelvin EP, McGavack TH, Kenigsberg S. Alpha-(2piperidyl) benzhydrol hydrochloride (pipradrol) as an adjunct in the dietary management of obesity. NY State J Med 1955; 55: 2336-2338

3 Tripod J, Sury E, Hoffmann K. Zentralerregende Wirkung eines neuen Piperidinderivates. Experientia 1954; 10: 261-262

4 European information system and database on new gruds drugs (EDND): https://ednd.emcdda.europa. eu (letzter Zugriff: 29.04.2016)

5 Davidson C, Ramsey J. Desoxypipradrol is more potent than cocaine on evoked dopamine efflux in the nucleus accumbens. J Psychopharmacol 2012; 26: $1036-1041$

6 Murray DB, Potts S, Haxton C et al. 'Ivory wave' toxicity in recreational drug users; integration of clinical and poisons information services to manage legal high poisoning. Clin Toxicol (Phila) 2012; 50 : 108-113

7 Iversen L, White M, Treble R. Designer psychostimulants: pharmacology and differences. Neuropharmacology 2014; 87: 59-65

8 Wood DM, Dargan PI. Use and acute toxicity associated with the novel psychoactive substances diphenylprolinol (D2PM) and desoxypipradrol (2DPMP). Clin Toxicol 2012; 50: 727-732

9 Corkery JM, Elliott S, Schifano F et al. 2-DPMP (desoxypipradrol, 2-benzhydrylpiperidine, 2-phenylmethylpiperidine) and D2PM (diphenyl2-pyrrolidin-2-yl-methanol, diphenylprolinol): A preliminary review. Prog Neuropharmacol Biol Psychiatry 2012; 39: 253-258
Hartmud Neurath

ist leitender Chemotechniker am Klinisch-toxikologischen Labor, Giftinformationszentrum Nord, Universitätsmedizin Göttingen. hneurath@

med.uni-goettingen.de

\section{Gesine Liebetrau}

ist Ärztin am Gemeinsamen Giftinformationszentrum, Erfurt.

liebetrau@ggiz-erfurt.de

\section{Simone Just}

ist Fachapothekerin am Gemeinsamen Giftinformationszentrum, Erfurt. just@ggiz-erfurt.de

\section{Dr. Maren}

Hermanns-Clausen

ist Oberärztin in der Klinik für Allgemein- und Jugendmedizin und Leiterin der Vergiftungs-Informations-Zentrale, Universitätsklinikum Freiburg. maren.hermanns-clausen@ uniklinik-freiburg.de

\section{Interessenkonflikt}

M.H.-C.: Einkünfte des Universitätsklinikums / der Vergiftungs-InformationsZentrale durch Dienstleistungsverträge mit Unternehmen der Chemischen Industrie und der Arzneimittel-Industrie. Teilnahme der Vergiftungs-InformationsZentrale an einem durch den Industrieverband Körperpflege und Waschmittel e. V. (IKW) mit finanzierten Forschungsprojekt der Gesellschaft für klinische Toxikologie (GfkT).

Die anderen Autoren geben an, dass kein Interessenkonflikt besteht.

DOI 10.1055/s-0042-107537

Dtsch Med Wochenschr 2016; 141: 951-953 (c) Georg Thieme Verlag KG . Stuttgart . New York . ISSN 0012-0472 